\title{
¿El valor de algunos servicios ecosistémicos de los Andes colombianos?: transferencia de beneficios por meta - análisis
}

\author{
Cesar Augusto Ruiz-Agudelo ${ }^{1 凶}$, Laura Carolina Bello
}

\author{
Valuation of the ecosystem services in the Colombian Andes. The benefit transfer \\ method: A meta-analysis
}

\begin{abstract}
The economic valuation of biodiversity and ecosystem services plays an important role in Colombia's conservation planning and economic development, but gathering data to conduct an original study can be expensive. To this end, there is an alternative yet controversial method called "benefits transfer". Here, we present a meta-analysis of available literature on the economic valuation of the Colombian Andes using two approaches: transfer functions and transfer of mean values. The economic value of ecosystem services, which included water availability, recreation and conservation for the Colombian Andes, yielded values between 106 and 339 trillion 2011 USD. However, determining the overall value of ecosystems presents a complex challenge due to the difficulty in the synthesis of the studies, their variability, and the nonexistence of documented experiences. Economic valuation in Colombia is faced with issues in the presentation, collection and variability of data. We concluded that the existing information is insufficient; there are few well-designed studies to procure consistent ecosystem values to support the creation of development policies in Colombia.
\end{abstract}

Keywords: Meta-analysis; meta-regressions; benefit transfer; valuation; ecosystem services; colombian Andes.

Edited by Alberto Acosta $\bowtie$

1. Conservación Internacional Colombia - Gerente Socioeconómico de Conservación Internacional Colombia.

2. Conservación Internacional Colombia

Received: 12-03-2014 Accepted: 10-05-2014

Published on line: 27-05-2014

Citation: Ruiz-Agudelo CA (2014) ¿El valor de algunos servicios ecosistémicos de los Andes colombianos?: transferencia de beneficios por meta-análisis. Universitas Scientiarum 19(3): 301-322

doi: 10.11144/Javeriana.SC19-3.vase

Funding: Conservación Internacional Colombia

Electronic supplementary material: $1-3$

\section{Introducción}

Colombia, a pesar de su reconocida diversidad natural, enfrenta el reto de alcanzar el desarrollo económico fundamentado en el manejo sustentable de sus recursos naturales y de los servicios que proveen los ecosistemas. ElPlanNacionaldeDesarrollo2010-2014 ("prosperidad para todos"), proyecta el crecimiento económico de la nación a través de la promoción de cinco locomotoras o motores de desarrollo (Agricultura, Minero-energético, Infraestructura, Vivienda e Innovación) que podrían afectar, en mayor o menor medida, la integridad del capital natural de Colombia, si las actividades que acompañan el proceso de desarrollo económico no contemplan el conocimiento y la gestión sostenible de la biodiversidad y sus servicios ecosistémicos. 
Recientemente, se ha evidenciado una creciente necesidad de incorporar, en las políticas de manejo y ordenamiento del territorio, el valor de los ecosistemas (De Groot et al. 2002, Scolozzi et al. 2012, Di Sabatino et al. 2013), buscando implementar acciones acertadas para el desarrollo socioeconómico de los territorios (Seppelt et al. 2012). Sin embargo, el conocimiento de los servicios ecosistémicos y del capital natural de Colombia sigue siendo disperso (Balbanera et al. 2012), no se cuenta con marcos metodológicos homogéneos que permitan usar esta información en la construcción de decisiones (Grunewald \& Bastian 2010, Bastian et al. 2012a, Bastian et al. 2012b, Seppelt et al. 2012, Van Oudenhoven et al. 2012, Bastian et al. 2013, Crossman et al. 2013), y su valor es pobremente entendido, generando que estos sean subvalorados por los mercados y los gobiernos (Nelson \& Daly 2010, Raudsepp-Hearne et al. 2010).

Una de las herramientas utilizadas, con la finalidad de corregir, es la valoración económica de la biodiversidad y de los servicios ecosistémicos (MA 2005, TEEB 2010, UK NEA 2011, Farley 2012). En los últimos años, un creciente cuerpo de literatura ha formulado los criterios necesarios para la producción de mapas de oferta y valor de los servicios ecosistémicos (Atkinson et al. 2012). En particular, la asignación de valores monetarios y la espacialización de los mismos, ha llegado a ser tema de investigación activa (Troy \& Wilson 2006, Maes et al. 2011a, Maes et al. 2011b, Ghermandi \& Nunes 2013, Schägner et al. 2013).

La asignación de valores monetarios requiere que estos sean relacionados con las áreas de provisión de los servicios (Farley 2012). Esto se puede realizar ya sea por el desarrollo de un nuevo estudio de valoración, o mediante la transferencia de valores a partir de estudios existentes para áreas similares (Navrud \& Bergland 2001, Navrud \& Ready 2007). La primera aproximación implica estimar el valor monetario de los servicios ecosistémicos en el área de estudio, a través de la aplicación de alguno de los métodos de valoración de mercado o no mercado disponibles. La segunda aproximación implica la transferencia de los valores calculados en uno o múltiples estudios previos, en donde el servicio ha sido valorado, al sitio de estudio (a menudo sitio denominado de la política).
Construir una aproximación precisa al valor de los servicios ecosistémicos de los Andes colombianos, a través de estudios puntuales y originales, es un proceso que configura un trabajo dispendioso y costoso, requiriendo el levantamiento de una gran cantidad de información para determinar el capital natural. Frente a esta dificultad existe una técnica conocida como transferencia de beneficios (Spash \&Vatn 2006, Plummer 2009), que permite aprovechar el esfuerzo realizado en estudios puntuales preexistentes, para construir una primera aproximación a la valoración en otras regiones o áreas similares, de forma rápida y económica (Brouwer 2000). Esta técnica consiste en la "adaptación de los valores monetarios de bienes ambientales estimados en una investigación original (sitio de estudio), a un contexto similar (sitio de la política), donde se desconoce el valor" (Rosenberger \& Loomis 2003, Osorio \& Correa 2004). La transferencia de beneficios tiene la ventaja de basarse en un meta - análisis de los resultados de los estudios realizados a manera de síntesis estadística, lo que permite: sintetizar la literatura de un tema particular, evaluar la hipótesis respecto a los efectos de las variables exploratorias en la construcción de los valores de interés, y usar el modelo estimado de meta - análisis para predecir valores estimados a través del tiempo y del espacio (Rosenberger \& Loomis 2001, Carriazo \& Ibañez 2003, Bergstrom \& Taylor 2006, Osorio 2006, Borenstein et al. 2009, Barrio \& Loureiro 2010).

Según Schägner et al. (2013) el 84\% de los estudios de valoración económica de servicios ecosistémicos publicados usan la transferencia de beneficios de forma total o parcial, debido a la practicidad de la técnica, su costo eficiencia y su potencial como herramienta para la síntesis de información dispersa (Woodward \& Wui 2001, Elsin et al. 2010, Liu et al. 2010, Barbier et al. 2011, Gascoigne et al. 2011, Brander et al. 2012, Christie \& Rayment 2012, De Groot et al. 2012, Brander et al. 2013, Ninan \& Inoue 2013, Schägner et al. 2013, Valdez et al. 2013).

El presente estudio realizó una transferencia de beneficios a partir de la aplicación de valores medios y funciones de meta-regresión, con el fin de tener una aproximación al valor y una visión de la distribución de parte del capital natural, en el contexto de los Andes Colombianos. La evaluación de los estudios permitió, analizar la calidad de la información disponible en 
Colombia (a la fecha de cierre de este estudio - año 2013), conocer los avances que ha desarrollado el país en esta materia, determinar los vacíos de información y aportar a la discusión sobre el futuro de la valoración económica de bienes y servicios ecosistémicos.

El estudio pretende responder a las preguntas: ¿qué valor representan los servicios que proveen los ecosistemas en los Andes colombianos?, ¿cuál es el estado del arte de la valoración económica de bienes y servicios ecosistémicos en el país?, ¿cómo se han adelantado los estudios de valoración económica de bienes y servicios ecosistémicos en Colombia?, ¿cuáles bienes y servicios se han valorado económicamente?, ¿qué técnicas se han usado en estos ejercicios de valoración?, ¿en cuales regiones de Colombia se han adelantado estudios?, ¿hay información suficiente, desde esta visión, para apoyar las decisiones de manejo ecosistémico en Colombia?, ¿cómo y para que, usar la información disponible?.

Los resultados presentados para la región de los Andes colombianos son discutidos a la luz de las limitaciones de la información disponible, presentando valores síntesis para algunos de los servicios ecosistémicos de la región, y formulando recomendaciones para el desarrollo de futuros estudios de valoración económica de bienes y servicios ecosistémicos en Colombia.

\section{Materiales y métodos}

La valoración de algunos de los servicios ecosistémicos en los Andes colombianos por medio del método de transferencia de beneficios contempló dos aproximaciones, la primera por transferencia de valores medios de las medidas de bienestar reportadas por los estudios originales (e.g. Disponibilidad a Pagar, Costos de Oportunidad, etc.) y la segunda por transferencia de funciones de meta - regresión de estas medidas de bienestar. Se siguieron los pasos propuestos en el Protocolo desarrollarlo por (Ruiz et al. 2011; Figura 1).

Paso uno: Definición del contexto del sitio de política: Se seleccionó a los Andes colombianos como el sitio de la política. Este se delimitó según los 14 Orobiomas del Mapa de Ecosistemas Continentales Marinos y Costeros de Colombia del IGAC (2010). Estos biomas se encuentran en 24 departamentos y 836 municipios del país (DANE 2005, DuqueEscobar 2007).

Paso dos: Selección bibliográfica de los estudios originales: Para la recolección de información de las investigaciones adelantadas a nivel nacional sobre valoración económica de biodiversidad, bienes y servicios ecosistémicos (el sitio de la Política), se utilizó el buscador ISI Web of Knowledge (Balvanera et al. 2012, Cardinale et al. 2012) con las

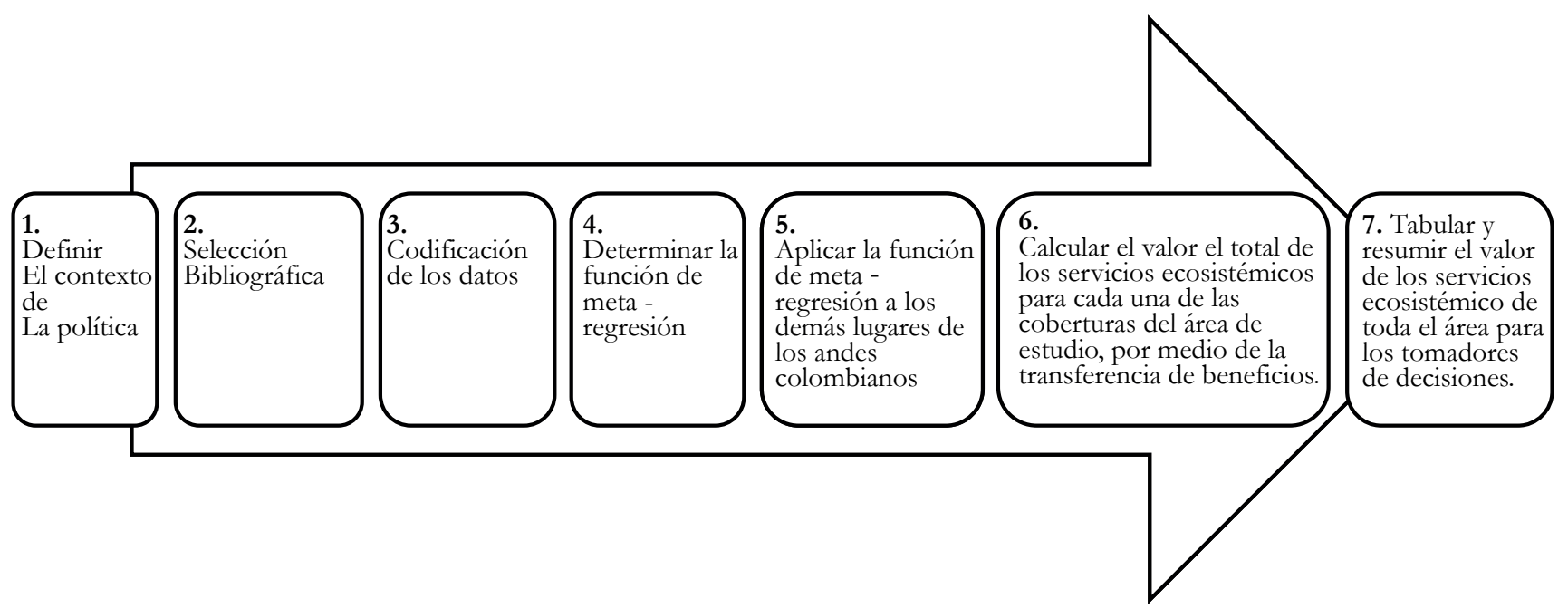

Fig. 1. Diagrama metodológico para realizar una transferencia de beneficios propuesto por Ruiz et al. (2011). 
palabras claves: "natural capital, ecosystem services, ecosystem value, economic value, capital natural, servicios ecosistémicos, strategy, framework, program, regional, national, Colombia". Se revisaron las bases de catálogos en línea de bibliotecas de universidades colombianas; al igual que documentos de políticas y textos técnicos. La búsqueda de información se completó visitando los centros de documentación de las 33 Corporaciones Autónomas Regionales de Colombia (CAR), se examinaron los dominios web de las principales ONG de conservación, y se utilizó el buscador genérico Google Scholar (Dec 1, 2013) (Tallis et al. 2008) empleando los términos anteriormente mencionados (Supt. 1).

Paso tres: Codificación de los datos o de las medidas de valoración económica recopiladas: De cada estudio (Figura 1) se recopilaron 41 variables de las sugeridas en el Protocolo de Ruiz-Agudelo et al. (2011), que se dividen en cinco tipos: a) variables del tipo de estudio, b) variables del tipo de servicio ecosistémico valorado, c) variables del método, d) variables del lugar y e) variables socioeconómicas (Supt. 2). Cada medida (e.g. Disponibilidad a Pagar -DAP, Costos de Oportunidad -COP, Costos de Viaje -CV, entre otras) fue clasificada según el servicio ecosistémico valorado y el método empleado con el fin de garantizar, una consistencia de los servicios evaluados y una consistencia en el tipo de medida, como lo sugieren Bergstrom \& Taylor (2006). La consistencia en las condiciones biofísicas y socio-ambientales, se analizó con dos niveles de profundidad:

1. Inicialmente se buscó una consistencia ajustada al clasificar las medidas según el tipo de bioma de los Andes donde se realizó el estudio, teniendo en cuenta esta clasificación y la del servicio ecosistémico valorado y el método implementado; generando así 55 bloques estadísticos con la información de las medidas de valoración reportadas en los estudios originales recopilados.

2. Posteriormente se realizó otra aproximación a la consistencia biofísica y socio-ambiental al considerar a los Andes colombianos como un área homogénea, con la finalidad de poder analizar el comportamiento de los valores estadísticos recopilados, y así contar con una mejor representatividad de la información disponible. De esta forma se conformaron 20 bloques de información estadística, según el tipo de método usado y el servicio ecosistémico valorado.

Se garantizó una consistencia temporal al homogenizar los valores monetarios reportados en cada estudio (valor del servicio, desviación estándar, ingreso promedio de la población), al valor presente de USD dólares de 2011; teniendo en cuenta la inflación anual y usando la tasa de cambio de $\$ 1793.47$ pesos colombianos por 1 dólar USD que se reportó (para Colombia) en Septiembre de 2011. De la misma forma, estas medidas fueron homogenizadas a la misma escala espacial, expresando el valor reportado por cada estudio, en dólares por hogar/mes y dólares por hectárea (ha)/mes. Esta transformación se realizó teniendo en cuenta el número de hogares y de hectáreas reportadas en cada uno de los estudios de referencia recopilados. Por último, los estudios fueron espacializados por medio de la asignación de una coordenada geográfica asociada al lugar donde se desarrolló.

Paso cuatro: Determinar y aplicar la función de meta - regresión más apropiada, de acuerdo a la información de base disponible, a los Andes colombianos: Se seleccionaron los estudios que presentaron información suficiente para ser incluidos en un meta-análisis (Figura 1), de acuerdo a Borenstein et al. (2009). De los 55 bloques generados para cada bioma especifico, ocho bloques fueron susceptibles de ser sometido a meta-análisis, ya que los estudios que los componen presentan más de dos valores y su respectivas varianzas (Tabla 1). De la misma forma, de los 20 bloques formados para los Andes como área homogénea, cinco fueron susceptibles de ser incluidos en el meta - análisis por presentar información completa, de acuerdo a los criterios metodológicos de Borenstein et al. (2009).

Para desarrollar el meta - análisis se eligió el modelo de valores aleatorios que reconoce que los estudios no son desarrollados con la misma metodología y por lo tanto los factores que afectan el valor son diferentes entre los estudios, reconociendo la variabilidad entre y dentro de los mismos (Borenstein et al. 2009). Bajo este modelo se computó el efecto promedio de los valores (M) por hogar/mes y ha/mes, y en los casos de 
Tabla 1. Bloques sometidos a meta-análisis considerando el tipo de bioma.

\begin{tabular}{lllll}
\hline \# & Bloque & Bioma & Método & Servicio \\
\hline 1 & OAA_DAP_DAGUA & Orobioma alto de los Andes & Valoración Contingente (DAP) & Disponibilidad de Agua \\
\hline 2 & OAA_DAP_RECREA & Orobioma alto de los Andes & Valoración Contingente (DAP) & Recreación \\
\hline 3 & OBA_CV_RECREA & Orobioma bajo de los Andes & Costo de Viaje & Recreación \\
4 & OBA_DAP_DAGUA & Orobioma bajo de los Andes & Valoración Contingente (DAP) & Disponibilidad de Agua \\
\hline 5 & OMA_CO_DAGUA & Orobioma medio de los Andes & Costo de Oportunidad & Disponibilidad de Agua \\
\hline 6 & OMA_DAP_CONSERVA & Orobioma medio de los Andes & Valoración Contingente (DAP) & $\begin{array}{l}\text { Conservación (valor de } \\
\text { legado y existencia) }\end{array}$ \\
\hline 7 & OMA_DAP_DAGUA & Orobioma medio de los Andes & Valoración Contingente (DAP) & Disponibilidad de Agua \\
\hline 8 & OMA_DAP_RECREA & Orobioma medio de los Andes & Valoración Contingente (DAP) & Recreación
\end{tabular}

la recreación se computó el efecto promedio M para el valor de la visita. Para este cómputo se le asignó un peso (Wi) a cada estudio según la suma de las dos variabilidades.

$$
\begin{aligned}
& \mathrm{M}=\frac{\sum_{(i=1)}^{\mathrm{k}} \mathrm{Wi} * \mathrm{Yi}}{\sum_{(i=1)}^{\mathrm{k}} \mathrm{Wi}} \\
& \mathrm{Wi}=\frac{1}{\mathrm{~V}_{\mathrm{Yi}}}
\end{aligned}
$$

De la misma forma se probó la hipótesis nula de que hay diferencias entre los efectos promedio, con un $95 \%$ de confianza. Una vez definido el efecto promedio (M), se evaluó la heterogeneidad de cada bloque por medio de las estadísticas $\mathrm{Q}, \mathrm{T}^{2}, \mathrm{I}^{2}$. La estadística Q refleja la tasa de variación observada en contraste con la esperada. $\mathrm{T}^{2}$ es la varianza del efecto promedio (M) o también entendida como la varianza entre los estudios, e $\mathrm{I}^{2}$ refleja la varianza que es explicada por diferentes condiciones entre los estudios y no solo por errores en las mediciones. Todos los cálculos fueron desarrollados según la metodología propuesta por Borenstein et al. (2009), en el lenguaje de programación $\mathrm{R}$.

$$
\mathrm{Q}=\sum_{(i=1)}^{\mathrm{k}} \mathrm{Wi}_{i} * \mathrm{Yi}^{2}-\frac{\left(\sum_{(i=1)}^{\mathrm{k}} \mathrm{WiYi}^{2}\right.}{\sum_{=1}^{\mathrm{k}} \mathrm{Wi}}
$$

$$
\begin{aligned}
& \mathrm{V}_{\mathrm{Yi}}=\mathrm{V}_{\mathrm{xi}}+\mathrm{T}^{2} \\
& \mathrm{~T}^{2}=\frac{\mathrm{Q}-\mathrm{df}}{\mathrm{C}} \\
& \mathrm{C}=\sum \mathrm{Wi}-\frac{\sum \mathrm{Wi}^{2}}{\sum \mathrm{Wi}} \\
& \mathrm{I}^{2}=\left(\frac{\mathrm{Q}-\mathrm{df}}{\mathrm{Q}}\right) \times 100 \%
\end{aligned}
$$

$$
\mathrm{df}=\mathrm{k}-1
$$

Donde: Vxi $=$ Varianza de cada estudio.

Se exploraron modelos para meta-regresión en aquellos bloques donde la varianza es explicada por diferentes condiciones entre los estudios y no solo por errores en las mediciones (I) y que tengan más de cuatro datos. Para obtener los modelos explicativos de las funciones de meta-regresión halladas, se usó la transformación $\log (\mathrm{x}+1)$ para todas las variables, se eliminaron los datos extremos fuertes y se realizó el análisis exploratorio basado en diez variables explicativas, por ser las que presentaban información completa (Borenstein et al. 2009). Ver descripción de cada variable con sus estadísticas descriptivas (Tabla 2).

La construcción de los modelos se realizó teniendo en cuenta todas las combinaciones posibles (de las variables explicativas) en cada bloque, contra las 
Tabla 2. Estadísticas descriptivas de las variables explicativas seleccionadas para la meta-regresión.

\begin{tabular}{|c|c|c|c|c|c|}
\hline Variable & Descripción & Promedio & Desv.est & Min & Max \\
\hline MSNM & Altitud & 2566.30 & 680.86 & 600 & 4000 \\
\hline ÁREA & Hectáreas en consideración & 15501.89 & 52156.02 & 6 & 306000 \\
\hline POBLA. LUGAR & Número de habitantes o visitantes & 451194.97 & 1504058.48 & 17.76 & 7000000 \\
\hline INGRESO & Ingreso promedio por individuo (IPC) & 4668.54 & 23044.52 & 104.26 & 144686.98 \\
\hline HOGAR & Número de hogares considerados & 95100.60 & 325180.33 & 45 & 1532870 \\
\hline DENS. HOGAR & Número de habitantes en el hogar & 5.32 & 2.20 & 1.50 & 14.69 \\
\hline EDAD & Promedio de edad & 1413.96 & 7156.92 & 0 & 37225 \\
\hline EDUCACIÓN & $\begin{array}{l}\text { Promedio de la educación (índice de 1-5) } \\
1 \text { - sin educación } \\
2 \text { - primaria } \\
3 \text { - secundaria } \\
4 \text { - tecnico } \\
5 \text { - profesional }\end{array}$ & 2.86 & 0.78 & 1.59 & 4.37 \\
\hline ESTRATO & Promedio del estrato (1-6) & 3.45 & 1.20 & 2.05 & 5 \\
\hline SEXO & Porcentaje de hombres & 0.62 & 0.09 & 0.47 & 0.86 \\
\hline MES_HOGAR & Dólar 2011/hogar/mes & 3054.29 & 14484.59 & 0.02 & 103378.92 \\
\hline HA_MES & Dólar 2011/ha/mes & 4882.68 & 23908.24 & 0.00 & 163110.95 \\
\hline VISITA & Dólar 2011/visita & 20.23 & 41.79 & 1.56 & 151.79 \\
\hline
\end{tabular}

variables respuesta de las medidas hogar/mes, ha/ mes, o visita. En total se realizaron 1367 modelos, de los cuales se analizaron 643 pertenecientes a los casos en que los bloques contenían más de cuatro medidas y el análisis de heterogeneidad demostró que la varianza observada es real y no reflejo de diferencias en la medición (Borenstein et al. 2009). Se eligió el modelo que presenta el mejor ajuste a los datos, teniendo en cuenta el criterio de información de Akaike (AICc) para muestras pequeñas. También se analizó la medida de ajuste $\mathrm{R}^{2}$ y la significancia de la regresión y los coeficientes. El $\mathrm{R}^{2}$ mide la proporción de la varianza explicada entre estudios. Este valor se obtiene a partir de la suma de cuadrados del error (SCE) y de la suma de cuadrados total (SCT), a partir de la ecuación:

$$
\mathrm{R}^{2}=1.0-\frac{\mathrm{SCE}}{\mathrm{SCT}}
$$

Donde: SCE es la suma de cuadrados de las distancias de los puntos a la curva de mejor ajuste, determinada por regresión. SCT es la suma de cuadrados de las distancias de los puntos desde una línea horizontal correspondiente a la media de todos los valores de $\mathrm{Y}$.

El criterio de información (AIC), combina la teoría de máxima verosimilitud y la entropía de información. Este criterio tiene en cuenta los cambios en la bondad de ajuste a partir de la función de la máxima verosimilitud, las diferencias en el número de parámetros entre dos modelos, y es definido por la siguiente ecuación:

$$
\mathrm{AIC}=-2 \times \log (\mathrm{lik})+2 \mathrm{~K}
$$

Donde: Lik es máxima verosimilitud. $\mathrm{K}$ es el número de parámetros.

Debido a los pocos estudios en cada bloque, se realizó la corrección para muestras pequeñas ya que presentan un mejor desempeño (Burnhan \& Anderson 2003).

$$
\mathrm{AICc}=\mathrm{AIC}+\frac{2 \mathrm{~K}(\mathrm{~K}+1)}{\mathrm{n}-\mathrm{K}-1}
$$


Este valor de AICc fue traducido en términos del peso que aporta cada modelo al bloque (Wi) y el radio de evidencia del ajuste (ERVI), el cual compara el AIC de un modelo analizado contra el AIC de otro modelo en competencia (Burnhan \& Anderson 2003).

$$
\text { ERVI.AIC }=\frac{\text { AIC del modelo }}{\text { AIC del siguiente mejor modelo }}
$$

El radio de evidencia se usó para determinar cuántas veces se desempeña mejor el modelo seleccionado frente a otro posible competidor (Burnhan \& Anderson 2003).

Paso cinco: Transferencias de beneficios: Se realizó una transferencia de valores medios para los nueve bloques, que contemplan la separación por Orobioma, en los que no fue posible desarrollar una meta - regresión. Se transfirió el valor del efecto promedio en cada caso. Se eligieron tres modelos que se transfirieron a los Andes colombianos, seleccionando uno para la Disponibilidad a Pagar por Agua, uno para la Disponibilidad a Pagar por Conservación y uno para la Disponibilidad a Pagar por Recreación, ya que son las medidas reportadas en los estudios originales. Las funciones de disponibilidad de agua y conservación se transfirieron a nivel de municipio, considerando los datos del Censo DANE (2005). Las funciones de recreación se transfirieron a todas las áreas protegidas de Colombia, utilizando el mapa de áreas protegidas de Vásquez \& Serrano (2009).

Pasos seis y siete: Aproximación a la valoración de algunos de los servicios ecosistémicos en los Andes colombianos: Para determinar el valor total de cada servicio evaluado para los Andes colombianos, se multiplicó los valores hallados por el área en hectáreas de cada Orobioma. En el caso de las funciones que se transfirieron a nivel de municipio, se multiplicó el valor hallado por el área en hectáreas de cada municipio. Finalmente, se desarrolló un análisis del porcentaje de error de cada valor transferido respecto a los valores de cada bloque, así:

$$
\% \mathrm{ERROR}=\frac{(\text { valor real }- \text { valor hallado })}{\text { valor real }}
$$

\section{Resultados y discusión}

Calidad y estado de la información sobre valoración económica, disponible en Colombia: Se recopilaron 58 estudios de valoración económica de servicios ecosistémicos desarrollados en los Orobimas de los Andes colombianos. De estos se obtuvieron 121 valores (o medidas económicas de valoración), ya que en algunos estudios se usó más de una metodología y se valoró más de un ecosistemas o un servicio.

El grupo de estudios compilado (Supt. 1) se caracterizó por su heterogeneidad, presentando vacíos en términos de su representatividad geográfica. De los 14 biomas de los Andes, en tan solo tres (Orobiomas alto, medio y bajo de los Andes, que suman el $86 \%$ del área de estudio) se encontraron estudios previos de valoración económica. El bioma con mejor información fue el Orobioma Medio de los Andes con más del 50\% de los estudios disponibles, seguido del Orobioma Bajo de los Andes (26\%) y el Orobioma Alto de los Andes (11\%) (Figura 2). En el 44\% de los departamentos andinos se reportó al menos un estudio. Cundinamarca presentó el mayor número con el 30\%, seguido por Boyacá, Antioquia y Santander. Otros departamentos con información de valoración económica, fueron: Nariño, Quindío, y Risaralda. La representatividad a escala de municipios, mostró estudios para el 4.48\% (Bogotá, Belmira y Encino, principalmente).

En la revisión se encontraron valoraciones de 22 servicios ecosistémicos. Observándose una mayor tendencia al desarrollo de estudios sobre disponibilidad de agua (oferta hídrica regional) y belleza escénica, desconociendo otros servicios culturales, e incluso los de provisión y regulación como la pesca, la madera, la leña, la polinización, el control biológico, entre otros. La mayoría de los servicios ecosistémicos evaluados en Colombia, reportan menos de cinco medidas; de hecho la mitad de los servicios valorados presentaron solo una medida, en muchos casos obtenida a través de transferencia de beneficios.

Se registró la aplicación de 13 métodos de valoración económica, el más usado fue la transferencia de beneficios, seguido de la valoración contingente con la medida de disponibilidad a pagar (DAP), el costo directo, el costo de viaje, el costo de oportunidad, 

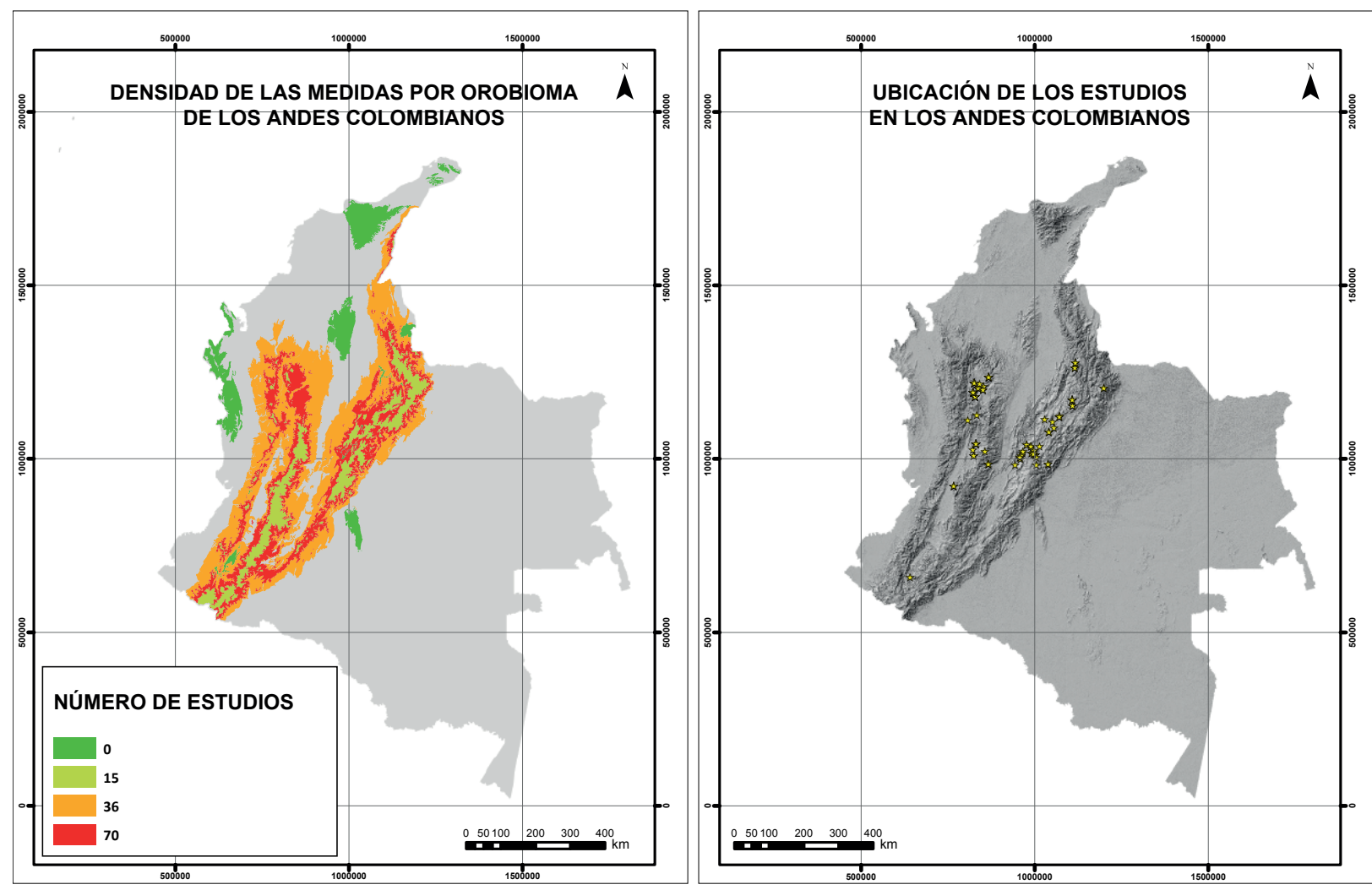

Fig. 2. Ubicación y representatividad de las medidas, en los biomas de los Andes de Colombia.

y los costos evitados. Las medidas calculadas por medio de transferencia de beneficios fueron descartadas para evitar transferir lo transferido. Debido a la variabilidad, no solo de los diferentes métodos usados, sino en la forma de aplicación de los mismos, se presentaron vacíos de información en las variables explicativas que son reportadas en cada estudio. Si bien la literatura considera variables centrales al aplicar cada método de valoración, estas no se encuentran en todos los estudios compilados, limitando la información susceptible de ser incorporada en los análisis de meta - regresión.

De las 95 variables explicativas halladas en los estudios disponibles para los Andes de Colombia, tan solo se consideraron diez, por ser las más comunes y las mejor soportadas (área, altitud -msnm, ingreso promedio per cápita, cantidad de hogares, edad, educación, sexo, estrato socioeconómico, densidad de habitantes por hogar y población afectada). La forma en que estas variables son presentadas en los estudios previos fue muy diversa, algunos generaron rangos, otros reportaron presencias-ausencias $(0 / 1)$ y otros el valor en bruto o el valor medio de la medida; razón por la cual se desarrolló un índice para homogeneizar cada variable. El área, la altitud (msnm), el número de hogares, la población del lugar, el ingreso, la educación y el sexo, presentaron los menores vacíos de información.

La determinación del valor global de los ecosistemas colombianos configura un reto complejo, debido a la dificultad en las síntesis de los estudios, la gran variabilidad de los mismos, y la falta de experiencias documentadas en valoración de servicios ecosistémicos. Si bien desde los años 90's se ha venido trabajando en la valoración de los servicios ecosistémicos del país, la mayoría de los esfuerzos de investigación se han desarrollado al nivel de trabajos de grado y consultorías, los cuales no son ampliamente difundidos ni publicados en revistas científicas indexadas (Seppelt et al. 2011). Por esta razón su conocimiento, análisis y validación fue un 
trabajo muy dispendioso, lo que hace posible que en este análisis hayan quedado excluidos algunos estudios desarrollados en Colombia.

Los estudios recopilados tienen el problema de la baja representatividad y la alta variabilidad, ya que las condiciones de los Andes colombianos son muy diversas; contando, bajo los mismos análisis, con estudios desarrollados en grandes ciudades y en poblaciones rurales pequeñas. Esta variabilidad de las condiciones de sitio se expresaron en valores extremos que debieron ser tratados con sumo cuidado, para reducir su influencia en la predicción; ya que transferencias de este tipo pueden no ser suficientemente precisas (Morrison \& Bergland 2006).

Los estudios revisados tienen como objetivos: innovaciones metodológicas, la formulación de un esquema de PSA (Pago Por Servicios Ambientales) o de alguna política de regulación, en los cuales la valoración es solo una etapa más del proceso de investigación. Es por esta razón, que no se reporta la información completa en los documentos disponibles. Por ejemplo, las estadísticas descriptivas, los detalles metodológicos y las características socioeconómicas de la población, rara vez son descritos en los estudios colombianos disponibles. Además, la definición de las variables es arbitraria (e.g. rangos) lo que dificulta el establecimiento de variables comunes para un meta - análisis. Futuros estudios deberán expresar una consistencia en las variables exploratorias, de tal forma que puedan ser comparables (Bergstrom \& Taylor 2006).

Contar un número reducido de estudios aumenta el riesgo de error y comparar estudios provenientes de lugares con características de sitio contrastantes, como las ciudades y las poblaciones rurales, aumenta la variabilidad de las condiciones de muestreo induciendo menor precisión (Morrison \& Bergland 2006, Eigenbrod et al. 2010). Los estudios puntuales solo representan las condiciones de un lugar específico, mas no las de toda la región; se ha demostrado que las relaciones presentes en los ecosistemas tienen variaciones geográficas importantes (Anderson et al. 2009, Eigenbrod et al. 2010).

Estos vacíos pueden inducir los tres errores planteados por Rosenberger \& Stanley (2006): Error de uniformidad, error de medición relacionado a la representatividad de la información (error de muestreo) y error de regionalización. Los errores de muestreo y de extrapolación de estudios provenientes de pequeñas regiones (errores de generalización), conducen reducciones adicionales en el poder de ajuste de los datos primarios compilados (Eigenbrod et al. 2010).

En la mayor parte de los estudios existentes en Colombia, se han dejado de abordar otras opciones de modelación de información, que pueden arrojar luces nuevas y certeras sobre la real provisión de servicios ecosistémicos, sus interrelaciones y retroalimentación (Nelson et al. 2009). En la actualidad, los estudios siguen aproximándose a los modelos de valoración por medio de funciones de regresiones logísticas, lineales o binomiales. Son pocos los estudios que usan otros tipos de modelación que tengan en cuenta las condiciones biofísicas como la cobertura, la hidrología, entre otras, y que son indispensables para el análisis de los problemas ecológicos complejos (McComb et al. 2006, Rosenberger \& Stanley 2006, Portman 2013).

Por las características de la información, descrita en los párrafos anteriores, es difícil desarrollar una transferencia de beneficios a partir de un modelo económico estricto como los modelos SUT (strong structural utility theoretic approach) o WSUT (weak structural utility theoretic approach) basados en la teoría de la utilidad, ya que para desarrollar estos modelos se necesitan las características socioeconómicas de los individuos, del servicio y de la información disponible, que en muchos casos no se reporta (Bergstrom \& Taylor 2006). El modelo de aproximación usado fue el modelo NSUT (Non-structural utility theoretic approach), el cual presenta variables exploratorias re lacionadas con la teoría económica, pero las conexiones entre estas variables y la subyacente función de utilidad no es explícitamente especificada. Este modelo tiene la ventaja de incorporar variables de múltiples fuentes de información científica que enriquecen el marco de formación de preferencias, y que de otra forma nunca serian tenidas en cuenta por el modelo estricto de la teoría de la utilidad (Spash \& Vatn 2006).

Meta - análisis. Bloques estadísticos diferenciados para cada tipo de bioma de los andes colombianos: La falta de información fue evidente cuando se buscó garantizar la consistencia espacial y se agruparon las 
medidas por bloques. El bloque compuesto por más medidas (OBA_DAP_DAGUA) solo tiene nueve datos, los demás no superan las cinco medidas (Tabla 1).

\section{Disponibilidad de agua - Oferta hídrica regional:} El meta - análisis de los bloques que valoraron la Disponibilidad de Agua mostró que el efecto promedio es mayor, cuando se aplica el método de costo de oportunidad y se hace la valoración por hectárea.

En los bloques que usan la Disponibilidad a Pagar (DAP) se observó que la aproximación al valor por este servicio es mayor en el Orobioma Medio de los Andes, seguido del Orobioma Bajo de los Andes y por último es menor en el Orobioma Alto; tanto para la valoración por hogares como por hectáreas (Tabla 3). Los valores resumen de la Disponibilidad a Pagar por bioma, oscilaron entre USD $\$ 0,43$ - USD $\$ 4,83$ dólares del 2011 por hogar al mes, y USD $\$ 0,06$ - USD $\$ 5,57$ dólares del 2011 por hectárea al mes. Los únicos casos donde la varianza entre los estudios fue real, son los que evaluaron los costos de oportunidad (I2=96.88), donde la variabilidad se pudo explicar en más de un $90 \%$ por factores de sitio; y en el caso de la Disponibilidad a Pagar por hectárea en el Orobioma Medio de los Andes (I2 hectárea=69.27), en donde la variabilidad se explicó en más de un $69 \%$ por condiciones del sitio. Lo anterior evidencia la heterogeneidad en la valoración y la producción de la tierra a lo largo de los Andes.

Recreación - belleza escénica: El meta - análisis de los bloques que valoraron el servicio de recreación mostró que el mayor efecto promedio de los estudios se presentó en el Orobioma Alto de los Andes, seguido del Orobioma Bajo, la menor valoración se otorgó en el Orobioma Medio. El efecto promedio de la Disponibilidad a Pagar (DAP) por recreación en el Orobioma Alto fue aproximadamente tres veces mayor que en el Orobioma Medio (Tabla 4). La varianza corresponde en más del 77\% a factores particulares de los sitios de los estudios. No se observaron diferencias significativas entre los valores otorgados por diferentes métodos usados, a pesar de sus diferencias conceptuales y supuestos metodológicos.

Conservación - Valor de legado y existencia: El meta - análisis del servicio ecosistémico opción de conservación, para el Orobioma Medio de los Andes evidenció, que el valor de Disponibilidad a Pagar (DAP) por hectárea fue más de tres mil veces mayor de lo que se está dispuesto a pagar por hogar. Estos valores por hectárea están influenciados por datos extremos provenientes de los estudios 12 y 13 (Supt. 1), desarrollados para los humedales de la ciudad de

Tabla 3. Efectos promedios en dólares del 2011 y sus estadísticas para la Disponibilidad de Agua. En negrilla bloque que se explica por condiciones de sitio.* Efecto estadísticamente significativo. $\mathbf{N}=$ número de estudios, $\mathbf{M}=$ valor resumen o efecto promedio, IC $\mathbf{9 5 \%}$ =intervalos de confianza con el $95 \%, \mathbf{P}=$ valor p prueba de hipótesis, $\mathbf{Q}=$ tasa de variación observada en contraste con la esperada. $\mathrm{T}^{2}=$ es la varianza del efecto promedio, $\mathrm{I}^{2}=$ porcentaje de variación explicada por factores de sitio.

Disponibilidad de agua - oferta

\section{Mes/Hogar}

\begin{tabular}{|c|c|c|c|c|c|c|c|}
\hline$\frac{\vec{\sigma}}{\overrightarrow{0}}$ & OAA_DAP_DAGUA & OBA_DAP_DAGUA & OMA_DAP_DAGUA & OAA_DAP_DAGUA & OBA_DAP_DAGUA & OMA_DAP_DAGUA & OAA_CO_DAGUA \\
\hline $\mathrm{n}$ & 3 & 9 & 4 & 3 & 9 & 4 & 2 \\
\hline M & 0.43 & 0.90 & 4.83 & 0.06 & 2.43 & 5.57 & 461.70 \\
\hline IC & $(0.26$ & $(-0.05 \mathrm{a}$ & $(1.93 \mathrm{a}$ & $(0.03 \mathrm{a}$ & $(0.17 \mathrm{a}$ & $(-6.73$ & $(37.8 \mathrm{a}$ \\
\hline $95 \%$ & a 0.58$)$ & 1.85) & 7.71) & $0.079)$ & 4.67) & a17.87) & 885.51) \\
\hline $\mathrm{P}$ & 0.68 & 0.98 & 0.85 & 0.67 & 1.00 & $0.02 *$ & $0 *$ \\
\hline Q & 0.78 & 1.89 & 0.81 & 0.81 & 0.86 & 9.76 & 32.01 \\
\hline $\mathrm{T}^{2}$ & 0 & 0 & 0 & 0 & 0 & 90.84 & 90680.83 \\
\hline $\mathrm{I}^{2}$ & 0 & 0 & 0 & 0 & 0 & 69.27 & 96.88 \\
\hline
\end{tabular}

\section{Hectárea/Mes}


Tabla 4. Efectos promedios en dólares del 2011 y sus estadísticas para la recreación por visita. ${ }^{*}$ Efecto estadísticamente significativo. $\mathbf{N}=$ número de estudios, $\mathbf{M}=$ valor resumen o efecto promedio, IC $\mathbf{9 5 \%} \mathbf{\%}=$ intervalos de confianza con el $95 \%, \mathbf{P}=$ valor p prueba de hipótesis, $\mathbf{Q}=$ tasa de variación observada en contraste con la esperada. $\mathbf{T}^{2}=$ es la varianza del efecto promedio, $\mathbf{I}^{2}=$ porcentaje de variación explicada por factores de sitio.

\begin{tabular}{|c|c|c|c|}
\hline \multicolumn{4}{|c|}{ Recreación - Visitas } \\
\hline Bloque & OAA_DAP_REC_ & OBA_CV_REC_ & OMA_DAP_REC \\
\hline $\mathrm{N}$ & 4 & 2 & 3 \\
\hline M & 10.049 & 8.88 & 3.79 \\
\hline IC $95 \%$ & (0.74 a 19.35) & $(-3.60$ a 21.38$)$ & $(-0.98$ a 8.57$)$ \\
\hline $\mathrm{P}$ & $0^{*}$ & $0.036^{*}$ & 0.123 \\
\hline Q & 66.489 & 4.402 & 4.188 \\
\hline $\mathrm{T}^{2}$ & 81.592 & 65.919 & 9.276 \\
\hline $\mathrm{I}^{2}$ & 95.488 & 77.282 & 52.242 \\
\hline
\end{tabular}

Bogotá, donde se tuvo en cuenta a toda la población de la capital (más de 7 millones de habitantes con aproximadamente un millón y medio de hogares) lo que hizo que la Disponibilidad a Pagar por hectárea fuese exageradamente alta. En este caso la varianza entre los estudios solo fue real en la valoración de las hectáreas, y se explica en un $86 \%$ por condiciones de sitio (Tabla 5).

Tabla 5. Efectos promedios en dólares del 2011 y sus estadísticas para la conservación por hectárea y hogar. * Efecto estadísticamente significativo. $\mathbf{N}=$ número de estudios, $\mathbf{M}=$ valor resumen o efecto promedio, IC $\mathbf{9 5} \%=$ =intervalos de confianza con el $95 \%, \mathbf{P}=$ valor $\mathrm{p}$ prueba de hipótesis, $\mathbf{Q}=$ tasa de variación observada en contraste con la esperada. $\mathbf{T}^{2}=$ es la varianza del efecto promedio, $\mathbf{I}^{2}=$ porcentaje de variación explicada por factores de sitio.

\begin{tabular}{|c|c|c|}
\hline \multicolumn{3}{|c|}{ Conservación } \\
\hline & Hectárea/Mes & Hogar/Mes \\
\hline Bloque & OMA_DAP_CO & OMA_DAP_CO \\
\hline $\mathrm{N}$ & 3 & 3 \\
\hline M & 13960.68 & 3.999 \\
\hline IC $95 \%$ & $(-11025.8$ a 38947$)$ & (2.01 a 5.98) \\
\hline $\mathrm{P}$ & $0.00^{*}$ & 0.60 \\
\hline Q & 14.97 & 1.03 \\
\hline $\mathrm{T}^{2}$ & $3.08 \mathrm{E}+08$ & 0 \\
\hline $\mathrm{I}^{2}$ & 86.64 & 0 \\
\hline
\end{tabular}

Bloques considerando a la región de los Andes colombianos, como un área homogénea

Disponibilidad de agua - Oferta hídrica regional: El análisis de los valores resumen de la disponibilidad de agua considerando a los Andes como un área homogénea, aumentó el número de estudios por bloque haciendo más contundente el análisis de significancia estadística $(p<0.05)$. El Costo de Oportunidad fue 20 veces mayor que la Disponibilidad a Pagar por hogar y 57 veces mayor que la Disponibilidad a Pagar por hectárea (Tabla 6). Los valores hallados por Disponibilidad a Pagar, con este enfoque, fueron muy similares a los del Orobioma Alto de los Andes (USD\$0,45 dólares del 2011/hogar/mes y USD \$1,75 dólares del 2011/hectárea/mes), la diferencia es que ahora los efectos promedio son estadísticamente significativos (diferentes a 0).

Recreación - Belleza escénica: Con este enfoque, para todos los casos, se confirmó la hipótesis de efecto resumen diferente a 0 . Las valoraciones promedio halladas, por medio del Costo de Viaje (CV), fueron casi el doble de lo que las personas están dispuestas a pagar por el servicio (DAP=USD \$8,06 dólares del 2011/visita; CV =USD \$15,68 dólares del 2011/visita). Los intervalos de confianza, aunque más reducidos que en los bloques por biomas, siguen siendo amplios (DAP=USD \$0,74 a USD \$19,35 dólares del 2011/visita; CV $=\mathrm{USD} \$ 1,85$ a USD $\$ 29,5$ dólares 2011/visita) evidenciando una amplia variabilidad entre los estudios originales (varianza explicada en un $89 \%$ por los factores del sitio). 
Tabla 6. Efectos promedios en dólares del 2011 y sus estadísticas para la disponibilidad de agua por hectárea y hogar. En negrilla el modelo seleccionado. $\mathbf{N}=$ número de estudios, $\mathbf{M}=$ valor resumen o efecto promedio, IC $\mathbf{9 5 \%} \%$ =intervalos de confianza con el $95 \%, \mathbf{P}=$ valor p prueba de hipótesis, $\mathbf{Q}=$ tasa de variación observada en contraste con la esperada. $\mathbf{T}^{2}=$ es la varianza del efecto promedio, $\mathbf{I}^{2}=$ porcentaje de variación explicada por factores de sitio.

\begin{tabular}{lllll}
\hline & & \multicolumn{2}{c}{ Disponibilidad de agua } \\
Bloque & DAP_DAGUA & CO_DAGUA & DAP_DAGUA & CO_DAGUA \\
\hline $\mathrm{N}$ & 16 & 2 & $\mathbf{1 6}$ & 2 \\
$\mathrm{M}$ & 0.45 & 8.921 & $\mathbf{1 . 0 7 5}$ & 461.702 \\
$\mathrm{IC}$ 95\% & $(0.27 \mathrm{a} 0.56)$ & $(0.64 \mathrm{a} 7.19)$ & $\mathbf{( 0 . 0 3 5}$ a 2.28) & $(37.8$ a 885.5$)$ \\
$\mathrm{P}$ & $0.00^{*}$ & $0.02^{*}$ & $\mathbf{0 . 0 4 0 *}$ & $0.02^{*}$ \\
$\mathrm{Q}$ & 5.28 & 19.34 & $\mathbf{1 7 . 3 9}$ & 32.01 \\
$\mathrm{~T}^{2}$ & 0 & 33.78 & $\mathbf{0 . 7 7}$ & 90680.83 \\
$\mathrm{I}^{2}$ & 0 & 94.83 & $\mathbf{1 3 . 7 6 9}$ & 96.88 \\
\hline
\end{tabular}

Conservación - Valor de legado y existencia: La valoración de la conservación de los Andes fue 55 veces mayor cuando se expresaron los valores por hectárea/ mes, en comparación a los valores por hogar/mes. En ambos casos las medidas fueron estadísticamente significativas (valor promedio hallado es diferente a $0)$. Solo en la valoración por hectárea, la variabilidad depende en un $81 \%$ de los factores del sitio $\left(I^{2}=81\right)$. En comparación con el análisis adelantado para cada uno de los biomas se observa, que el sesgo provocado por los estudios de la ciudad de Bogotá (estudios 12 y 13 Supt. 1) se reduce cambiando el valor de USD $\$ 13.960,68$ a USD \$223,14 dólares del 2011 por hectárea.

Funciones de meta - regresión halladas para la disponibilidad a pagar por oferta hídrica, en los Andes colombianos: Para hallar las funciones que explican la Disponibilidad a Pagar en los Andes colombianos, se analizó el bloque de DAP_DAGUA (ha/mes) para los Andes homogéneos. De los 511 modelos probados, los que mostraron un mejor desempeño a la luz de la línea base nacional, fueron los que consideran a la edad y al sexo.

$$
\begin{aligned}
& \text { Disponibilidad a }\left(\frac{\text { ha }}{\text { mes }}\right)=0.306 \log (\text { edad }+1)+0.056^{1} \\
& \text { 1. p intercepto }=0.883 \text {, p_ edad }=0.014 \\
& \begin{array}{l}
\text { Disponibilidad a } \\
\text { pagar por agua }
\end{array}\left(\frac{\text { ha }}{\text { mes }}\right)=-37.00 \log (\text { sexo }+1)+18.586^{2} \\
& \text { 2. } \text { p intercepto }=0.010 \text {, p_sexo }=0.014
\end{aligned}
$$

El modelo que explica la Disponibilidad a Pagar por hectárea en función de la edad fue el más parsimonioso (AICC $=17,84)$ seguido de cerca por el que considera el sexo (AICC $=17,913)$. Estos recogen el 29\% de los pesos relativos de todos los modelos $(\mathrm{Wi}=0,148+0,142)$ y explican el $43 \%$ de la varianza de los datos $\left(\mathrm{R}^{2}\right.$; Tabla 7).

La relación de la edad y el sexo con la Disponibilidad a Pagar por agua por ha/mes, mostró una correlación negativa con el sexo $(\mathrm{Rs}=-0,8)$ y positiva pero menor con la edad ( $R s=0,33)$, lo que indica que a menor cantidad de hombres la disponibilidad a pagar es mayor, es decir las mujeres están más dispuestas a pagar por el servicio, sobre todo si tienen mayor edad.

Funciones de meta - regresión, halladas para la recreación (belleza escénica) en los Andes colombianos: Para hallar las funciones que explican la variabilidad de las medidas relacionadas con la recreación, se realizaron meta - regresiones para la Disponibilidad a Pagar por recreación para los Andes homogéneos (DAP_REC). En total se probaron 73 modelos, las variables explicativas que se incluyeron, fueron la altura (msnm), el área, el ingreso y la población del lugar.

Las funciones de meta - regresión halladas para el servicio de belleza escénica mostraron que los modelos que explican la valoración en términos del área o la altitud (msnm), son los más parsimoniosos. Estos modelos compiten muy de cerca (ya que el 
Tabla 7. Estadísticas de desempeño de modelos hallados para la DAP por disponibilidad de Agua. Se presentan 13 casos de 511 a manera de comparación. En negrilla el modelo seleccionado. $\mathbf{n}=$ número de estudios, $\mathbf{p}=$ significancia estadística *modelo significativo con $\boldsymbol{\alpha}=0.05, \mathbf{K}=$ número de parámetros involucrados, AICC=criterio Akaike con corrección a muestras pequeñas, $\mathbf{W} \mathbf{i}=$ peso relativo del modelo, Eviratio=radio de evidencia de desempeño del modelo seleccionado frente a los demás modelos.

\begin{tabular}{lllllllll}
\hline Modelo & $\mathbf{n}$ & $\mathbf{K}$ & $\mathbf{p}$ & $\mathbf{R}^{2}$ & $\mathbf{R}^{2}$ adjust & AICC & Wi & Eviratio \\
\hline EDAD ha_mes & $\mathbf{1 3}$ & $\mathbf{2}$ & $\mathbf{0 . 0 1 4}$ & $\mathbf{0 . 4 3 9}$ & $\mathbf{0 . 3 8 8}$ & $\mathbf{1 7 . 8 4 1}$ & $\mathbf{0 . 1 4 8}$ & \\
SEXO ha_mes & $\mathbf{1 3}$ & $\mathbf{2}$ & $\mathbf{0 . 0 1 4}$ & $\mathbf{0 . 4 3 6}$ & $\mathbf{0 . 3 8 4}$ & $\mathbf{1 7 . 9 1 3}$ & $\mathbf{0 . 1 4 2}$ & $\mathbf{1 . 0 3 7}$ \\
DENSHOGAR EDAD ha_mes & 13 & $\mathbf{3}$ & 0.018 & 0.519 & 0.423 & 20.164 & 0.046 & 3.196 \\
INGRESO EDAD ha_mes & 13 & 3 & 0.219 & 0.517 & 0.421 & 20.220 & 0.045 & 3.286 \\
DENSHOGAR SEXO ha_mes & 13 & 3 & 0.019 & 0.507 & 0.408 & 20.500 & 0.039 & 3.780 \\
INGRESO SEXO ha_mes & 13 & 3 & 0.228 & 0.497 & 0.396 & 20.752 & 0.034 & 4.286 \\
MSNM SEXO ha_mes & 13 & 3 & 0.602 & 0.458 & 0.350 & 21.719 & 0.021 & 6.954 \\
MSNM EDAD ha_mes & 13 & 3 & 0.604 & 0.451 & 0.342 & 21.878 & 0.020 & 7.137 \\
HOGAR EDAD ha_mes & 13 & 3 & 0.744 & 0.451 & 0.341 & 21.888 & 0.020 & 7.566 \\
HOGAR SEXO ha_mes & 13 & 3 & 0.744 & 0.451 & 0.341 & 21.895 & 0.019 & 7.594 \\
POBLALUGAR EDAD ha_mes & 13 & 3 & 0.649 & 0.450 & 0.341 & 21.902 & 0.019 & 7.619 \\
\hline POBLALUGAR SEXO ha_mes & 13 & 3 & 0.649 & 0.450 & 0.340 & 21.902 & 0.019 & 7.620 \\
\hline EDAD SEXO ha_mes & 13 & 3 & 0.019 & 0.439 & 0.327 & 22.174 & 0.017 & 7.679 \\
\hline
\end{tabular}

área es tan solo 1,9 veces mejor) con el que considera la altitud, y son más de un millón de veces mejores que el modelo que considera la relación entre las dos variables (Eviratio $=1$ '244.671,243). Los dos modelos recogen el 100\% del peso relativo (Tabla 8).
Se evidencia que la disponibilidad a pagar depende del área, como se presenta a continuación:

Disponibilidad a

pagar por visita $=0.085 \log \left(\right.$ Area+1) $+1.371^{1}$

1. P_área $=0.212$ no es significativo. P_intercepto $=0.034$ es significativo.

Tabla 8. Estadísticas de desempeño de modelos hallados para la DAP por recreación. Se presentan ocho casos de 31 a manera de comparación. En negrilla el modelo seleccionado. $\mathbf{n}=$ número de estudios, $\mathbf{p}=$ significancia estadística *modelo significativo con $\boldsymbol{\alpha}=0.05, \mathbf{K}=$ número de parámetros involucrados, $\mathrm{AICC}=$ criterio Akaike con corrección a muestras pequeñas, $\mathbf{W i}=$ peso relativo del modelo, Eviratio=radio de evidencia de desempeño del modelo seleccionado frente a los demás modelos.

\begin{tabular}{lllllllll}
\hline Modelo & $\mathbf{n}$ & $\mathbf{K}$ & $\mathbf{p}$ & $\mathbf{R}^{2}$ & $\mathbf{R}^{2}$ adjust & AICC & Wi & Eviratio \\
\hline ÁREA VISITA & $\mathbf{6}$ & $\mathbf{2}$ & $\mathbf{0 . 2 1 2}$ & $\mathbf{0 . 3 5 4}$ & $\mathbf{0 . 1 9 3}$ & $\mathbf{2 5 . 3 5 2}$ & $\mathbf{0 . 6 6 3}$ & \\
MSNM VISITA & 6 & 2 & 0.386 & 0.191 & -0.011 & 26.705 & 0.337 & 1.967 \\
MSNM ÁREA VISITA & 6 & 3 & 0.349 & 0.532 & 0.220 & 53.421 & 0.000 & 1244671.243 \\
POBLALUGAR VISITA & 5 & 2 & 0.551 & 0.201 & -0.198 & Inf & 0.000 & Inf \\
\hline
\end{tabular}


La relación de la Disponibilidad a Pagar por visita con el área es positiva $(\mathrm{Rs}=0.60)$, indicando que a media que el área que brinda el servicio de recreación es mayor, la Disponibilidad a Pagar por el acceso a ella es mayor. De la misma forma, la relación con la altitud (msnm) es positiva (Rs=0.42), indicando que si el área se encuentra a mayor altitud mayor es la Disponibilidad de Pagar para acceder a la zona.

Funciones de meta - regresión halladas para la conservación (valor de legado y valor de existencia) en los Andes colombianos: Los modelos hallados para la Disponibilidad a Pagar por conservar, contemplaron las variables explicativas altitud (msnm), área, población del lugar, número de hogares y densidad del hogar. Los que presentaron el mejor desempeño fueron los que explican la Disponibilidad a Pagar por conservar, en términos de las variables relacionadas con la cantidad de habitantes o usuarios potenciales de la conservación. De hecho este modelo acumula más del 40\% de los pesos (Wi). El modelo que contempla el número de hogares del lugar, tiene un despeño apenas 3,04 veces mejor que el segundo que contempla la altitud (Eviratio; Tabla 9). A la luz de la información analizada la Disponibilidad a Pagar por conservar, se puede expresar como:

$\begin{aligned} & \text { Disponibilidad a } \\ & \text { pagar por conservar }\end{aligned}=0.845 \log ($ hogar +1$)-3.293^{*}(17)$

*p_hogar $=0.079, \mathrm{P}_{\text {_ intercepto }}=0.45$ no son significativo.
Se observa una relación positiva con la población (Rs= 0,798), a mayor concentración de población mayor es la Disponibilidad a pagar por conservar, y una relación negativa con la altitud $(\mathrm{Rs}=-0,667)$, cuando las personas se encuentran a menor altitud (msnm) tienen mayor Disponibilidad a Pagar por conservar, que las que están en las partes altas de los Andes colombianos.

Aproximación a la valoración económica de algunos servicios ecosistémicos de los Andes colombianos, por transferencia de valores medios: Se realizó una transferencia de valores medios para los nueve bloques en los que fue posible desarrollar un meta - análisis, transfiriendo el valor del efecto promedio en cada caso. Se encontró que las Disponibilidades a Pagar por agua en las partes media y baja (de los Andes) son mayores a las presentadas en las partes altas, probablemente relacionado a que en las partes altas la población disfruta directamente de un servicio de buena calidad y en abundancia, mientras que las personas en las partes bajas sufren los efectos de contaminación y escases. Se debe considerar el efecto que las grandes ciudades ubicadas en las partes medias de los Andes, generan en la medida de Disponibilidad a Pagar (por concentrar mayor función de demanda declarada). En las grandes ciudades los habitantes tienen mayor Disponibilidad a Pagar, debido a la mayor dependencia del servicio. A pesar de esta mayor Disponibilidad a Pagar por disponibilidad de agua en la parte media de los Andes, su monto (declarado) no

Tabla 9. Estadísticas de desempeño de modelos hallados para la Disponibilidad a Pagar por conservar. Se presentan seis casos de 103 a manera de ilustración del ejercicio. En negrilla el modelo seleccionado. $\mathbf{n}=$ número de estudios, $\mathrm{p}=$ significancia estadística $*$ modelo significativo $\operatorname{con} \boldsymbol{\alpha}=0.05, \mathbf{K}=$ número de parámetros involucrados, $\mathrm{AICC}=$ criterio Akaike con corrección a muestras pequeñas, $\mathbf{W} \mathbf{i}=$ =peso relativo del modelo, Eviratio=radio de evidencia de desempeño del modelo seleccionado frente a los demás modelos.

\begin{tabular}{lllllllll}
\hline Modelo & $\mathbf{n}$ & $\mathbf{K}$ & $\mathbf{p}$ & $\mathbf{R}^{2}$ & $\mathbf{R}^{2}$ adjust & AICC & Wi & Eviratio \\
\hline HOGAR ha_mes & $\mathbf{6}$ & $\mathbf{2}$ & $\mathbf{0 . 0 7 9}$ & $\mathbf{0 . 5 7 9}$ & $\mathbf{0 . 4 7 4}$ & 46.691 & $\mathbf{0 . 4 4 4}$ & \\
MSNM ha_mes & 6 & 2 & 0.187 & 0.387 & 0.234 & 48.943 & 0.144 & 3.084 \\
DENSHOGAR ha_mes & 6 & 2 & 0.732 & 0.033 & -0.209 & 51.684 & 0.037 & 12.138 \\
AREA ha_mes & 6 & 2 & 0.945 & 0.001 & -0.248 & 51.874 & 0.033 & 13.351 \\
SEXO ha_mes & 5 & 2 & 0.129 & 0.590 & 0.453 & 54.688 & 0.008 & 54.509 \\
MSNM HOGAR ha_mes & 6 & 3 & 0.008 & 0.970 & 0.950 & 60.832 & 0.000 & 1176.676 \\
\hline
\end{tabular}


alcanza a compensar el Costo de Oportunidad que tiene una hectárea en las partes altas para proveer el servicio (CO $=$ USD $\$ 461,702$ dólares del 2011/ha vs $-\mathrm{DAP}=\mathrm{USD} \$ 2,42+$ 0,5 dólares del 2011/ha).

La recreación (belleza escénica) se comporta diferente. La Disponibilidad a Pagar es mayor en el Orobioma Alto Andino, lo que está relacionado con el imaginario de conservación que se tiene sobre los bosques alto andinos y páramos de Colombia.

El análisis de la conservación refleja, que esta empaqueta bajo su nombre todo un conjunto de beneficios incluyendo valores de existencia y legado. Se observa que (a la luz de la línea base nacional para los Andes) se está más dispuesto a pagar por conservar lugares altos, debido a la cantidad de servicios esenciales que potencialmente proveen.

$\mathrm{Al}$ reconocer la relación de dependencia entre la prestación de los servicios por los Orobiomas de las partes altas y el aprovechamiento de los mismos en las partes medias y bajas, donde se concentra la mayoría de la población, se observa que el monto de la Disponibilidad a Pagar por conservación de los habitantes del Orobioma Medio cubre los costos de oportunidad de los Orobiomas Altos. Lo que podría ser un buen indicativo para la promoción de futuras políticas de conservación entre estos Orobiomas.

El valor total de los servicios ecosistémicos (que se pudieron abordar en este estudio) para los Orobiomas de los Andes, por el método de transferencia de valores medios asciende a USD \$105.775'974.313,69 dólares del 2011. El valor de la disponibilidad de agua en los Andes asciende a USD \$76 $501.474,83$ dólares del 2011, el de recreación asciende a USD \$70664.446,7 dólares del 2011, y el valor que se está dispuesto a pagar por conservación (las comunidades del Orobioma Medio de los Andes) tiene un monto de USD \$105.628`808.392,20 dólares del 2011.Considerando solamente los servicios ecosistémicos estrictos (Agua y Recreación), se tiene que el valor de estos corresponde a USD\$147`165.921,49 dólares del 2011.

El mayor error de transferencia está en el Costo de Oportunidad por hectárea (1024.2\%) seguido del mes (558.3\%), los demás errores no superan el 100\%. El porcentaje de error está relacionado con la cantidad de datos que se consideraron y la dispersión de los mismos.
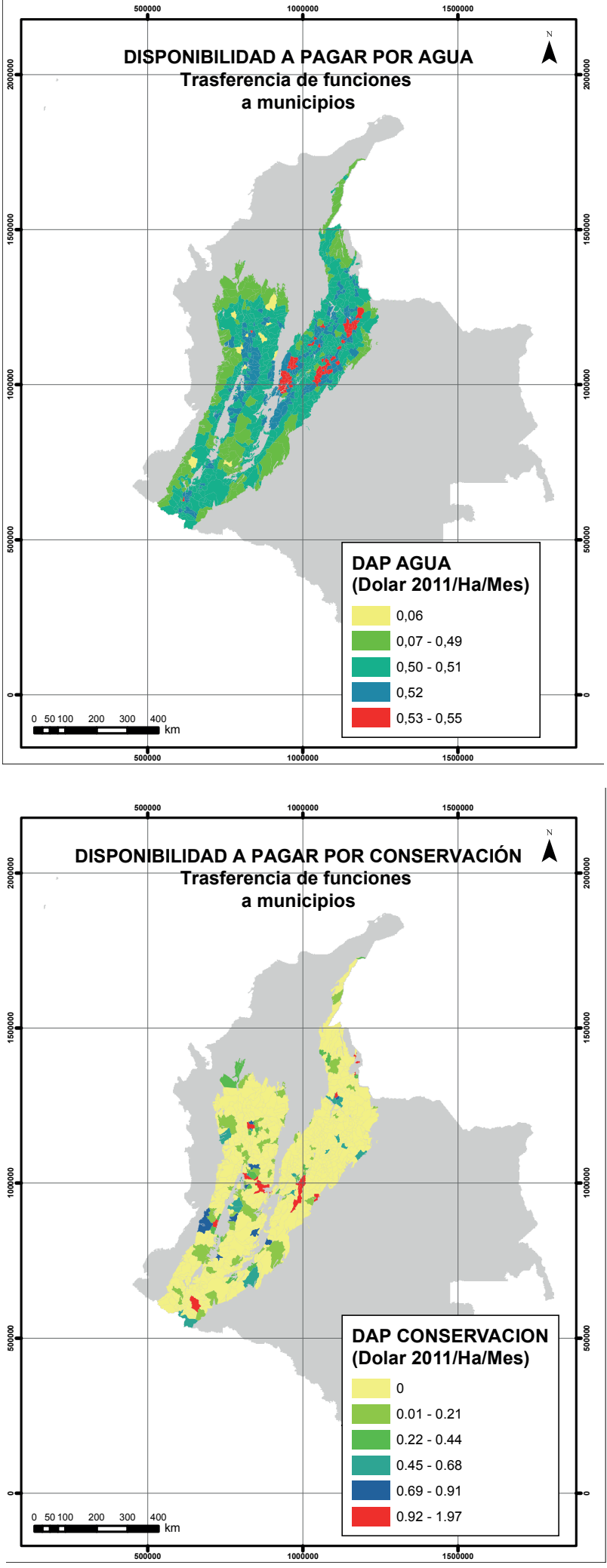

Fig. 3. Valoración de los Andes colombianos según las funciones aplicadas a los municipios. a. Disponibilidad a Pagar por agua. b. Disponibilidad a Pagar por Conservación. 
Aproximación a la valoración económica de algunos servicios ecosistémicos de los Andes colombianos, por transferencia de funciones: $\mathrm{Al}$ aplicar la transferencia de las funciones seleccionadas, se observa: 1) - La Disponibilidad a Pagar por la Disponibilidad de Agua es muy variable a lo largo de los municipios de los Andes, concentrando los mayores valores en la cordillera oriental (Figura 3). 2) -La mayor Disponibilidad a Pagar por conservación se concentran en las grandes ciudades (Bogotá, Medellín, Pasto, Ibagué, Bucaramanga, Cúcuta, Cali, Manizales). 3) - Las funciones para la recreación fueron transferidas a las áreas protegidas de Colombia, ya que es en estas áreas donde se concentra el servicio de belleza escénica. Los valores muestran una mayor valoración de las áreas protegidas de orden nacional, sobre las regionales y locales. Esta transferencia de funciones arrojó un valor total que asciende a USD \$338.937’632.975,54 dólares del 2011. El mayor aporte es de la Disponibilidad a Pagar por la conservación, mientras que la Disponibilidad a Pagar por agua asciende a USD\$1.190`115.047, 11 dólares del 2011.

Los errores hallados en las funciones de meta regresión son más altos que los hallados por el valor medio (DAP agua 187.73\%; DAP conservación $101.93 \%$, DAP recreación 74\%, CV recreación $67.58 \%$ ); esto responde a la significancia de las ecuaciones halladas, influenciada por la restricción del número de estudios, la variabilidad de los mismos y la cantidad de información disponible en las variables explicativas.

Medidas para controlar los tipos de error de la transferencia de beneficios, para los Andes colombianos: Los tipos de error fueron compensados a través de la forma en que se planteó el bloqueo estadístico de la información. El error de regionalización fue controlado al considerar solo los estudios desarrollados en ecosistemas de los Andes colombianos, no se incluyeron estudios provenientes de otros países andinos. Al controlar las diferencias entre los biomas se redujo la variabilidad atribuible a las características socioeconómicas y biofísicas propias de los sitios de estudio, versus los sitios de política.

El error de medición (error de muestreo) se restringió, al analizar con precaución los datos atípicos provenientes de estudios realizados en lugares particularmente diferentes. Se generaron bloques de estudios que aplicaron el mismo método de valoración, esta separación evitó comparar medidas provenientes de diferentes funciones de utilidad (Bergstrom \& Taylor 2006). Sin embargo, al interior de cada método de valoración aplicado existen otros factores metodológicos o decisiones a juicio de cada investigador que no son reportados de forma exhaustiva en los estudios disponibles, por lo que el error sigue siendo importante (Rosenberger \& Stanley 2006). Johnston et al. (2006) concluyen que estos errores se pueden eliminar con más estudios, o identificando las asunciones metodológicas realmente correctas, sin embargo es más plausible la primera opción.

La aplicación de la transferencia de beneficios en los Andes colombianos: La aplicación de la transferencia de beneficios para los Andes colombianos tuvo tres propósitos: 1) resumir y evaluar los estudios de valoración adelantados en el país, 2) determinar variables que influencian los valores reportados, y 3) aplicar predicciones basadas en modelos meta - analíticos. Cada uno de estos propósitos está condicionado por el grado de precisión y el nivel de información con que se cuente, ya que "la transferencia puede ser tan exacta como los estudios primarios" (Brookshire \& Neill 1992, Wilson \& Hoehn 2006). Por lo tanto, el nivel predictivo de la presente transferencia de beneficios se ve comprometido. Este estudio genera una aproximación para entender los patrones de valor otorgados a algunos de los servicios ecosistémicos a lo largo de los Andes colombianos, haciendo una comparación entre los resultados obtenidos por diferentes métodos.

Este análisis consideró a los Andes como un sistema heterogéneo e influenciado por las variaciones altitudinales y bióticas que condicionan las prácticas y las características socio - culturales. Por ejemplo, se consideraron las diferencias entre un agricultor de los páramos colombianos (Orobioma alto de los Andes) cuyo sistema productivo predominante es la papa y cebolla, y un agricultor de tierras bajas (Orobioma bajo de los Andes) cuyo sistema productivo predominante es café, frutales, caña, etc. Se comprendió la diversidad de los ecosistemas y de las comunidades de los Andes, buscando la mayor similitud entre los sitios de los estudios y los sitios de las políticas, generando medidas más ajustadas (Bockstael et al. 2000). 
La transferencia se realizó de manera gruesa con los valores hallados por el efecto resumen del meta análisis de cada bloque, mostrando un valor continuo a modo de generalización que se debe entender como una medida indicativa de bienestar ajustada a regiones biogeográficas comparables entre ellas, asumiendo un error de uniformidad (Rosenberger \& Stanley 2006).

Las diferencias en los órdenes de magnitud de los valores, revelaron que el mayor bienestar asociado a la disponibilidad de agua se encuentra en el Orobioma Medio de los Andes, donde el requerimiento del recurso enfrenta las mayores presiones por densidades demográficas y donde la orografía de altas pendientes no permite la acumulación del mismo. Los valores revelados muestran una desigualdad en el mercado al compararlos con el costo de oportunidad de una hectárea en el Orobioma Alto de los Andes, de donde proviene el servicio, ya que este no alcanza a ser compensado por la Disponibilidad a Pagar proveniente de Orobiomas de menor altitud.

Esta aparente desigualdad del mercado puede ser subsidiada al reconocer que una hectárea no presta un solo servicio, sino que su conservación representa una provisión de múltiples servicios asociados. En otras palabras, la conservación de una hectárea de determinado Orobioma aporta a una gran variedad de servicios ecosistémicos. Cuando se analiza la medida de bienestar reflejada en la Disponibilidad por Conservar una hectárea, se observa que el valor revelado es mucho mayor reflejando que, en el imaginario del individuo, la conservación de un ecosistema es altamente valorada por que comprende una gran variedad de beneficios e interrelaciones.

Se identificaron los sitios que concentran la oferta y la demanda de los servicios, y las variables que influyen fuertemente en la variabilidad de estos valores (Naidoo et al. 2008, Eigenbrod et al. 2010). El uso de modelos sensibles espacialmente al sitio de los estudios, generó estimaciones agregadas que revelaron de mejor forma la variabilidad espacial de las transferencias por valores medios; permitiendo la identificación de áreas prioritarias (Bateman et al. 2006, Eigenbrod et al. 2010).

Los modelos hallados se alimentaron con la agrupación de información disponible considerando a los Andes colombianos como un área homogénea, y aunque se esperaba una mejora por el incremento en el número de estudios por bloque, se presentaron mayores errores de transferencia por este enfoque que por el anterior (diferenciando los biomas andinos). Sin embargo, esta aproximación brindó luces para entender la variabilidad que tienen las medidas de valoración a través de sistemas heterogéneos como los Andes colombianos.

A partir de esta transferencia se generó una imagen espacial de la distribución de los valores. Estos mapas son de gran importancia ya que, al conocer la concentración de la oferta y la demanda, se pueden plantear estrategias para el mantenimiento de servicios ecosistémicos clave (Naidoo et al. 2008). Debido a la ausencia de datos primarios en la mayoría de los lugares, estos mapas de distribución de servicios ecosistémicos son una buena aproximación. La variabilidad espacial en los mapas de distribución, responde a las variables que explican las medidas de valoración. En la mayoría de los modelos se encontró una baja significancia estadística en las variables predictivas, esta situación debe ser entendida como un reflejo de la deficiencia de los datos y no como una falta de efecto (Smith 1992, Walsh et al. 1992, Smith \& Huang 1995, Bowland \& Beghin 2001).

Variables socioeconómicas como el sexo, la edad y la población, influyen fuertemente en los modelos hallados para la valoración de la disponibilidad de agua y la conservación. La variable ingreso no fue determinante en los análisis, debido a que en la mayoría de los estudios este dato se aproxima al salario mínimo vigente; bajo esta similitud, otras variables (como las demográficas) fueron determinantes. La edad y el sexo revelaron que las mujeres y las personas adultas tienen mayor Disponibilidad a Pagar.

La densidad de la población explica la Disponibilidad a Pagar por conservación, al punto que la mayor proporción se centró en las grandes ciudades. Reflejando el potencial de desarrollar políticas de compensación para ecosistemas estratégicos, a partir de esta disponibilidad. Las variables físicas como la altitud y el área son las que condicionan la valoración de los servicios de recreación, evidenciando su dependencia a los atributos del lugar y no a las condiciones socioeconómicas. 
Recomendaciones para el desarrollo de futuros estudios devaloracióneconómicadelabiodiversidad y los servicios ecosistémicos en Colombia (Supt. 3): Esta contribución formula recomendaciones frente a los requerimientos de información necesarios para adelantar mejor transferencia de beneficios, de tal forma que futuros estudios puedan ser aprovechados al máximo garantizando los tres tipos de consistencia necesaria para reducir el error (Johnston et al. 2006). La recomendación principal busca, la creación de una base de datos en línea que contenga la información de estudios de valoración de los ecosistemas de Colombia, permitiendo la estandarización de la información compilada mediante la aplicación de protocolos metodológicos estrictos (Supt. 3). Iniciativas como esta se han desarrollado en otros países mostrando buenos resultados, por ejemplo el ERVI "Environmental Valuation Reference Inventory" que cuenta hoy con cerca de 1500 estudios (ERVI 1997); la base de datos "Envalue data base" cuenta con 400 estudios (NSW EPA 1995); la base "Ecosystem Services" que cuenta con cerca de 300 estudios (Natural Capital Project 2012) y la base de datos "Review of Externality" con 200 estudios (Institute of Studies for the Integration of Systems 2002). La creación de estas bases de información ha permitido, que el acceso a los datos en bruto alimente modelos específicos para regiones puntuales a partir de datos agrupados, reduciendo así el error de regionalización.

Con una plataforma nacional que compile sistemáticamente las investigaciones en valoración económica, se busca que la información sea comparable y robusta, facilitando el desarrollo de transferencias con modelos mejor ajustados y resultados fiables que puedan ser considerados en la formulación de las políticas socioeconómicas. Además, se aporta a la construcción de estudios en múltiples contextos con una mejor comprensión de los sistemas tropicales, y de las preferencias de los usuarios en países en vías de desarrollo (McComb et al. 2006).

Loomis \& Rosenberger (2006) proponen que los reportes de información sean alentados por incentivos institucionales, bancos de datos, premios anuales para estudios de políticas pertinentes, entre otros, de tal forma que los investigadores y editores busquen publicar su información considerando la posibilidad de una futura transferencia de beneficios. En otras palabras "El pensamiento creativo es necesario para poner en práctica los incentivos que estimulen la generación de datos empíricos suministrando la materia prima necesaria para la transferencia de beneficios" (Wilson \& Hoehn 2006).

El uso de la transferencia de beneficios para la valoración económica de la biodiversidad, los bienes y los servicios ecosistémicos debe ser un área en la que los economistas y otros investigadores se muevan con cuidado, pero no deben permitir que su cautela inhiba estos esfuerzos por completo. La mejor solución de valoración siempre será la recopilación y el uso de información primaria, con datos específicos del sitio. A falta de los recursos necesarios para ello, cuidadosas transferencias pueden proporcionar información veraz sobre los valores de los ecosistemas (Johnston et al. 2006, Plummer 2009).

\section{Conclusión}

La aproximación al valor económico de algunos de los servicios ecosistémicos en los Andes colombianos, arrojó valores entre USD \$106 a USD \$339 mil millones de dólares del 2011, que aún son una subestimación del valor económico total ya que no considera toda la gama de servicios ecosistémicos provistos por los Andes colombianos. La variabilidad de estos valores evidencia que la elección de diferentes métodos, para hacer la transferencia, tiene un efecto significativo en el valor estimado. A través del método de transferencia de funciones se halló mayor valoración que por el método de valores medios; siendo evidente, que al considerar la variabilidad espacial se obtiene una valoración más puntual de los servicios ecosistémicos. Este tipo de análisis proporciona una perspectiva diferente sobre las razones por las cuales la transferencia de beneficios parece tan controversial, y plantea preocupaciones sobre las limitaciones de los trabajos de aquellos que apoyan este tipo de aplicaciones como método de valoración formal de la biodiversidad, los bienes y los servicios ecosistémicos.

\section{Agradecimientos}

Los autores deseamos expresar nuestro agradecimiento a Conservación Internacional Colombia, especialmente a Jose Vicente Rodriguez Mahecha (Director Científico) y Fabio Arjona Hincapié (Director Ejecutivo), por la financiación 
y apoyo del presente estudio. Además a los colegas: Dra. Angela Maria Cortes Gómez, Dr. Francisco Madriñan, Dr. Andres Páez por sus revisiones y comentarios.

\section{Conflicto de intereses}

Los autores expresan que no existe conflicto de intereses.

\section{Referencias}

Anderson BJ, Armsworth PR, Eigenbrod F, Thomas CD, Gillings S, et al. (2009) Spatial covariance between biodiversity y other ecosystem service priorities. Journal of Applied Ecology 46:888-896

Atkinson G, Bateman I, Mourato S (2012) Recent advances in the valuation of ecosystem services and biodiversity. Oxford Review of Economic Policy 28(1):22-47 doi:10.1093/oxrep/grs007

Balvanera P, Uriarte M, Almeida-Lenero L, Altesor A, DeClerk F, et al. (2012) Ecosystem services research in Latin America: the state of the art. Ecosystem Services 2:56-70

Barbier EB, Hacker SD, Kennedy C, Koch EW, Stier AC "et al"(2011) The value of estuarine and coastal ecosystem services. Ecological Monographs 81(2):169-193

Barrio M,Loureiro ML (2010) A meta-analysis of contingent valuation forest studies. Ecological Economics 69:10231030 doi:10.1016/j.ecolecon.2009.11.016

Bastian O, Haase D, Grunewald K (2012a) Ecosystem properties, potentials and services-the EPPS conceptual framework and an urban application example. Ecological Indicators 21:7-16

Bastian O, Grunewald K, Syrbe RU (2012b) Space and time aspects of ecosystem services, using the example of the EU Water Framework Directive. International Journal of Biodiversity Science, Ecosystem Services and Management 8(1):5-16 http://dx. doi.org/10.108 $0 / 21513732.2011 .631941$

Bastian O, Syrbe RU, Rosenberg M, Rahe D, Grunewald K (2013) The five pillar EPPS framework for quantifying, mapping and managing ecosystem services. Ecosystem Services 4:15-24 http://dx.doi.org/10.1016/j. ecoser.2013.04.003

Bateman IJ, Day B, Georgiou S, Lake I (2006) The aggregation of environmental benefit values: Welfare measures, distance decay and total WTP. Ecological Economics 60(2):450-460

Bergstrom JC, Taylor LO (2006) Using meta-analysis for benefits transfer: Theory and practice. Ecological Economics 60:351-360
Bockstael N, Freeman III AM, Kopp R, et al. (2000) On measuring the economic values for nature. Environmental Science Technology 34:1384-89

Borenstein M, Hedges LV, Higgins J, Rothstein H (2009) Introduction to Meta-analysis. United Kingdon, Wiley Publications

Bowland B, Beghin J (2001) Robust estimates of value of a statistical life for developing economies. Journal of Policy Modeling 23:386-396

Brander L, Brouwer R, WagtendonK A (2013) Economic valuation of regulating services provided by wetlands in agricultural landscapes: A meta-analysis. Ecological Engineering 56:89-96 http://dx.doi.org/10.1016/j. ecoleng.2012.12.104

Brander LM, Wagtendonk AJ, Hussain S, McVittie A, Verburg PH, et al. (2012) Ecosystem service values for mangroves in Southeast Asia: A meta-analysis and value transfer application. Ecosystem Services 1:62-69 http://dx.doi.org/10.1016/j.ecoser.2012.06.003

Brookshire DS, Neill HR (1992) Benefit transfers conceptual y empirical issues. Water Resources Research 28(3):651-655

Brouwer R (2000) Environmental value transfer: state of the art and future prospects. Ecological Economics 32:137-152

Burnhan K, Anderson D (2003) Model selection y multimodel inference. A practical InformationTheoretic Approach. Second Edition Springer, New York

Cardinale BJ, Duffy JM, Gonzalez A, Hooper DU, PerringsCh, et al. (2012) Biodiversity loss and its impact on humanity. Science 486(11148):59-67

Carriazo F, Ibáñez AM (2003) Valoración de los beneficios económicos provistos por el Sistema de Parques Nacionales Naturales: una aplicación del análisis de transferencia de beneficios. Documento CEDE, Universidad de los Andes, Bogotá

Christie M, Rayment M (2012) An economic assessment of the ecosystem service benefits derived from the SSSI biodiversity conservation policy in England and Wales. Ecosystem Services 1:70-84 http://dx.doi. org/10.1016/j.ecoser.2012.07.004

Crossman ND, Burkhard B, Nedkov S, Willemen L, Petz $\mathrm{K}$, et al. (2013) blueprint for mapping and modeling ecosystem services. Ecosystem Services 4:4-14 http:/ / dx.doi.org/10.1016/j.ecoser.2013.02.001

DANE (2005) Censo General. Perfil Colombia. Departamento Administrativo Nacional de Estadística. Colombia. http:www.dane.gov. cofilescenso2005perfilesperfil_nal.pdf 
De Groot RS, Brander LM, Vander Ploeg S, Costanza R, Bernard F, et al. (2012) Global estimates of the value of ecosystems and their services in monetary units. Ecosystem Services 1:50-61 http://dx.doi. org/10.1016/j.ecoser.2012.07.005

De Groot RS, Wilson MA, Boumans RMJ (2002) A typology for the classification, description and valuation of ecosystem functions, goods and services. Ecological Economics 41:393-408

Di Sabatino A, Coscieme L, Vignini P, Cicolani B (2013) Scale and ecological dependence of ecosystem services evaluation: Spatial extension and economic value of freshwater ecosystems in Italy. Ecological Indicators 32:259-263 http://dx.doi.org/10.1016/j. ecolind.2013.03.034

Duque-Escobar G (2007) Aspectos geofísicos de los andes de Colombia. Desafío de la población de los andes.1er Congreso Internacional de Desempeño Humano en Altura. Noviembre 22 de 2007. Manizales- Colombia. Disponible en: http:www.galeon. comgeomecanicaalturas.htm

Eigenbrod FP, Armsworth R, Anderson BJ, Heinemeyer A, Gillings S, et al. (2010) Error propagation associated with benefits transfer-based mapping of ecosystem services. Biological Conservation 143:2487-2493

Elsin YK, Kramer RA, Jenkins WA (2010)Valuing Drinking Water Provision as an Ecosystem Service in the Neuse River Basin. Journal of water resources planning and management July/august 2010 doi:10.1061/_ASCE_ WR.1943-5452.0000058

ERVI (1997) "Environmental Valuation Reference Inventory" develop by Civita, P, Filion F, Frehx J, Jay.M. Disponible en www.ervi.ca

Farley J (2012) Ecosystem services: The economics debate. Ecosystem Services 1:40-49

Gascoigne WR, Hoag D, KoontzL, Tangen BA, Shaffer TL, et al. (2011) Valuing ecosystem and economic services across land-use scenarios in the Prairie Pothole Region of the Dakotas, USA. Ecological Economics 70:17151725 doi:10.1016/j.ecolecon.2011.04.010

Ghermandi A, Nunes PALD (2013) A global map of coastal recreation values: Results from a spatially explicit metaanalysis. Ecological Economics 86:1-15 http://dx.doi. org/10.1016/j.ecolecon.2012.11.006

Grunewald K, Bastian O (2010) Ökosystem dienstleistun genanalysieren - begriff-licherundkon zeptioneller Rahmenaus lands chaftsökologischer Sicht. GEOÖKO 31:50-82

IGAC (2010) Ecosistemas continentales, costeros y marinos de Colombia. Escala 1:500000. Proyecto "mejora de los sistemas de cartografía del territorio colombiano". República de Colombia
Institute of Studies for the Integration of Systems (2002) Review of Externality Data.RED. European Commission, Research Directorate, Bruselas. Disponible en: http:www.isis-it.netred

Johnston RJ, Besedin EY, Ranson MH (2006) Characterizing the effects of valuation methodology in function based benefits transfer. Ecological Economics 60(2):407-419

Liu S, Costanza R, Troy A, D'Aagostino J, Mates W (2010) Valuing New Jersey's Ecosystem Services and Natural Capital: A Spatially Explicit Benefit Transfer Approach. Environmental Management 45:1271-1285 doi 10.1007/s00267-010-9483-5

Loomis JB, Rosenberger RS (2006) Reducing barriers in future benefit transfers: Needed improvements in primary study design y reporting. Ecological Economics 60(2):343-350

Maes J, Braat L (2011a) A Spatial Assessment of Ecosystem Services in Europe: Methods, Case Studies and Policy Analysis-Phase 1, Ispra, Italy: Partnership for European Environmental Research

Maes J, Paracchini ML, Zulian G (2011b) A European Assessment of the Provision ofEcosystem Services. Towards an Atlas of Ecosystem Services, Luxembourg

Millennium Ecosystem Assessment MA (2005) Ecosystems and Human Well- being: Synthesis. Island Press, Washington. D.C.

McComb G, Lantz V, Nash K, Rittmaster R (2006) International valuation databases: Overview, methods y operational issues. Ecological Economics 60(2):461-472

Morrison M, Bergland O (2006) Prospects for the use of choice modelling for benefit transfer. Ecological Economics 60(2):420-428

Naidoo R, Balmford A, Costanza R, Fisher B, Green RE, et al. (2008) Global mapping of ecosystem services y conservation priorities. Proceedings of the National Academy of Sciences of the United States of America 105:9495-9500

Natural Capital Project (2012) Environmental Services Project Database. Stanford University's Woods Institute for the Environment, University of Minnesota's Institute on the Environment, The Nature Conservancy, World Wildlife Fund. Disponible en http:www.naturalcapitalproject.orgdatabase.html

Navrud S, Bergland O (2001) In: Spash CL, Carter C (ed) Value Transfer and Environmental Policy. University of Cambridge, Cambridge

Navrud S, Ready R (2007) Review of methods for value transfer. In: Navrud S, Ready R (ed) Environmental Value Transfer: Issues and Methods. The Economics of Non-Market Goods and Resources. Springer, Netherlands, pp 1-10 
Nelson EJ, Daly G (2010) Modelling ecosystem services in terrestrial systems. F1000 Biology Reports 2:53

Nelson EJ, Mendoza G, Regetz J, Polasky S, Tallis HT, et al. (2009) Modeling multiple ecosystem services, biodiversity conservation, commodity production, and tradeoffs at landscape scales. Frontiers in Ecology and the Environment 7:4-11

Ninan KN, Inoue M (2013) Valuing forest ecosystem services: Case study of a forest reserve in Japan. Ecosystem Services 5:78-87 http://dx.doi. org/10.1016/j.ecoser.2013.02.006

NSW EPA (1995) The ENVALUE environmental valuation database. Deparment of Environment and Climate Change, Australian Government. Disponible en: http:www.environment.nsw.gov.auenvalueapp

Osorio JD (2006) El método de transferencia de beneficios para la Valoración económica de servicios ambientales: Estado del arte y aplicaciones. Semestre Económico 9:107-12

Osorio JD, Correa F (2004) Valoración económica de costos ambientales: Marco conceptual y métodos de estimación. Semestre Económico 13:159-193

Plummer ML (2009) Assessing benefit transfer for the valuation of ecosystem services. Frontiers in ecology and the environment 7(1):38-45 doi: 10.1890/080091

Portman M (2013) Ecosystem services in practice: Challenges to real world implementation of ecosystem services across multiple landscapes - A critical review. Applied Geography 45:185-192 http://dx.doi. org/10.1016/j.apgeog.2013.09.011

Raudsepp-Hearne C, Peterson GD, Tengö M, Bennett EM, Holland T, et al. (2010) Untangling the Environmentalist's Paradox: Why Is Human Well-being Increasing as Ecosystem Services Degrade?. BioScience 60(8):576-589

Rosenberger RS, Loomis JB (2001) Benefit Transfer of Outdoor Recreation Use Values: A technical document supporting the Forest Service Strategic Plan 2000 revision. F.S. Department of Agriculture, Rocky Mountain Research Station, Gen. Tech. Rep. RMRSGTR-72. Fort Collins, CO: U.S, pp 59

Rosenberger RS, Loomis JB (2003) Benefit transfer. In: Champ PA, Boyle KJ, Brown TC (ed) A Primer on Nonmarket Valuation, Kluwer, Dordrecht, The Netherlands

Rosenberger RS, Stanley TD (2006) Measurement, generalization y publication: Sources of error in benefits transfer y management. Ecological Economics 60:372-378

Ruiz-Agudelo CA, Bello C, Londoño-Murcia MC, Alterio H, Urbina- Cardona JN, et al. (2011) Protocolo para la valoración económica de los servicios ecosistémicos en los Andes colombianos, a través del método de transferencia de beneficios. Reflexiones sobre el Capital Natural de Colombia No. 1. Conservación Internacional Colombia.Bogotá,D.C,pp53.https:// docs.google.com/ file/d/0BwvbL6AbT4QXd3EyWE9pNFBMVTQ/ edit. ISBN: 978-958-99731-4-1

Schägner JP, Brander L, Maes J, Hartje J (2013) Mapping ecosystem services' values: Current practice and future prospects. Ecosystem Services 4:33-46 http://dx.doi. org/10.1016/j.ecoser.2013.02.003

Scolozzi R, Morri E, Santolini R (2012) Delphi-based change assessment in ecosystem service values to support strategic spatial planning in Italian landscapes. Ecological Indicators 21:134-144 http://dx.doi. org/10.1016/j.ecolind.2011.07.019

Seppelt R, Dorman C, Eppink F, Lautenbach S, Schmidt S (2011) A quantitative review of ecosystem service studies: approaches, shortcomings y the road ahead. Journal of applied Ecology 48:630-636

Seppelt R, Fath B, Burkhard B, Fisher JL, GrêtRegamey A, et al. (2012) Form follows function? Proposing a blue print for ecosystem service assessments based on reviews and cases studies. Ecological Indicators 21:145-154

Smith VK (1992) On separating defensible benefit transfers from "smoke and mirrors". Water Resources Research 28:85-94

Smith VK, Huang JC (1995) Can markets value air quality? A hedonic meta-analysis of hedonic property value models. Journal of Political Economy 103(1):209-227

Spash CL, VatnA (2006) Transferring environmental value estimates: Issues and alternatives. Ecological Economics 60:379-388 doi:10.1016/j.ecolecon.2006.06.010

Tallis HT, Kareiva P, Marvier M, Chang A (2008) An ecosystem services framework to support both practical conservation and economic development. Proceedings of the National Academy of Sciences 105(28):9457-9464

The Economics of Ecosystems \& Biodiversity TEEB (2010) The Economics of Ecosystems and Biodiversity: Ecological and Economic Foundations. London, Earths can, Washington D.C.

Troy A, Wilson MA (2006) Mapping ecosystem services: practical challenges and opportunities in linking GIS and value transfer. Ecological Economics 60(2):435-449

UK NEA (2011) UK National Ecosystem Assessment: Understanding Nature's Value to Society. Synthesis of the Key Findings, Cambridge

Valdez VC, Ruiz-Luna A, Ghermandi A, Nunes PALD (2013) Valuation of ecosystem services provided by coastal wetlands in northwest Mexico. Ocean \& Coastal Management 78:1-11 http://dx.doi.org/10.1016/j. ocecoaman.2013.02.017 
Van Oudenhoven APE, Petz K, Alkemade R, Hein L, De Groot RS (2012) Framework for systematic indicator selection to assess effects of land management on ecosystem services. Ecological Indicators 21:110-122

Vásquez VH, Serrano M (2009) Las áreas naturales protegidas de Colombia. Conservación InternacionalFundacion Biocolombia, Bogotá, pp 696

Walsh RG, Johnson DM, McKean JR (1992) Benefit transfer of outdoor recreation demand studies, 19681988. Water Resources Research 28(3):707-713

¿El valor de algunos servicios ecosistémicos de los Andes colombianos?: transferencia de beneficios por meta-análisis

Resumen. La valoración económica de la biodiversidad y los servicios ecosistémicos es importante para la planificación del desarrollo socioeconómico de Colombia. La obtención de datos primarios es costosa, pero existe un método denominado "transferencia de beneficios". Se presenta una aproximación a la valoración de algunos servicios ecosistémicos en los Andes colombianos incorporando un meta-análisis de los estudios realizados en Colombia, aplicando: transferencia de funciones y transferencia de valores medios. El valor de los servicios ecosistémicos (disponibilidad de agua, recreación y conservación), arrojó valores entre 106 a 339 mil millones de dólares en 2011. La determinación del valor global de los ecosistemas configura un reto complejo, debido a la dificultad en la síntesis de los estudios, la variabilidad de los mismos, y la ausencia de experiencias documentadas. La valoración económica en Colombia, enfrenta el inconveniente de la presentación, obtención y variabilidad de datos. Se concluyó que la información existente es insuficiente, encontrándose pocos estudios bien diseñados. Esta contribución formula recomendaciones para futuros ejercicios.

Palabras clave: Meta-análisis; meta-regresiones; transferencia de beneficios; valoración; servicios ecosistémicos; Andes colombianos.
Wilson M, Hoehn JP (2006) Valuing environmental goods y services using benefit transfer: The state of the art y science. Ecological Economics 60(2):335-342

Woodward RT, Wui YS (2001) The economic value of wetland services: a meta-analysis. Ecological Economics $37: 257-270$

¿O valor de alguns serviços ecossistémicos dos Andes colombianos?: transferência de benefícios por meta-análise

Resumo. A valorização económica da biodiversidade e os serviços ecossistémicos é importante para a planificação do desenvolvimento socioeconómico de Colômbia. A obtenção dos dados primários utiliza um método denominado "transferência de benefícios". Apresenta-se uma aproximação da avaliação de alguns serviços ecossistémicos nos Andes colombianos incorporando uma meta-análise dos estudos realizados em Colômbia, aplicando: transferência de funções e transferência de valores médios. $\mathrm{O}$ valor dos serviços ecossistémicos (disponibilidade de água, recreação e conservação), exibiu valores entre 106 a 339 mil milhões de dólares em 2011. A determinação do valor global dos ecossistemas configura um desafio complexo, devido à dificuldade na síntese dos estudos, a variabilidade dos mesmos, e a ausência de experiências documentadas. A avaliação económica em Colômbia, enfrenta o inconveniente da presentação, obtenção e variabilidade de dados. Concluiuse que a informação existente é insuficiente, encontrando-se poucos estudos bem desenhados. Esta contribuição formula recomendações para futuros exercícios.

Palavras-chave: Meta-análise; meta-regressões; transferência de benefícios; avaliação; serviços ecossistémicos; Andes colombianos. 